\title{
GEOGRAFÍA COMERCIAL APLICADA Y POLÍTICA URBANA: METODOLOGÍA Y RESULTADOS EN EL CASO DE TRES CANTOS (MADRID)
}

\author{
POR \\ ANTONIO MORENO JIMÉNEZ
}

\section{Introducción}

La emersión y expansión de las nuevas formas de instalaciones comerciales (centros comerciales, hipermercados, grandes superficies especializadas, etc.) encuentra su entorno más propicio en las áreas metropolitanas por razones harto conocidas. El pequeño comercio se ve sometido allí a una feroz competencia y afronta dificultades de todo orden, bien para resistir, bien para nacer. Estos retos y cambios en esa función económica no dejan insensibles a los municipios de las coronas metropolitanas que se enfrentan a opciones y dilemas importantes: a veces han optado por maridarse con potentes empresas de distribución y facilitar la ubicación en su término de esas nuevas formaciones comerciales periféricas que, entre otros impactos, trasbocan la movilidad y el transporte, los hábitos de consumo o los flujos de gasto; en otras ocasiones han debido atender a los intereses del sector comercial detallista instalado para impedir o paliar su deterioro, lo que acarrearía secuelas indeseables en el empleo, las rentas urbanas, los cambios de uso del suelo, etc. Sea como fuere, la cuestión

Antonio Moreno Jiménez. Departamento de Geografía. Universidad Autónoma de Madrid. 
siempre posee múltiples facetas que geográficamente han sido estudiadas: económica, urbanistica, social, ambiental, etc.

La toma de conciencia por parte de los responsables municipales $\mathrm{y}$ autonómicos de la enjundia de esos cambios ha motivado la promoción de estudios técnicos, tendentes sobre todo a diagnosticar la situación y establecer las politicas comerciales urbanas posibles, en diversas regiones y áreas metropolitanas españolas (vid. Moreno, 1995, p. 124-6). El caso que aquí se expondrá se inserta dentro de esas coordenadas y versa sobre el municipio madrileño de Tres Cantos. Aparte del interés que el caso concreto puede suscitar, por tratarse de una ciudad planificada «ex novo», con este artículo se desea divulgar una experiencia de geografía comercial aplicada que trata de entroncar, no sólo con la proyección más operativa que esta parcela de la disciplina posee en muchos otros paises (vid. Jones y Simmons, 1990; Merenne-Schoumaker y Browet, 1996; Birkin et al., 1996; Longley y Clarke, 1995), sino también con la orientación hacia una mayor profesionalización de los geógrafos en un terreno, como el del «geomarketing» (Beaumont, 1991), apenas explotado en España y con notables posibilidades para la aplicación del saber geográfico. A tal fin en el apartado siguiente se realiza una somera presentación del contexto geográfico y de los síntomas que movieron al Ayuntamiento a encargar un estudio de geografía comercial aplicada ${ }^{1}$. Seguidamente se resumen los principales hallazgos acerca de los dos bloques temáticos investigados (la oferta y la demanda), para finalizar con una síntesis de las propuestas y recomendaciones.

El contexto geográfico de la ciudad y los sintomas negativos en su sistema comercial

Tres Cantos es una ciudad nueva de magnitud medio-pequeña (casi 28.000 hab. según el padrón de 1996), situada en una posición privilegiada: dista $21 \mathrm{~km}$. del centro de Madrid, con el que se comu-

1 El presente articulo se apoya en un extenso informe titulado «El equipamiento comercial y la evasión del poder de compra de los consumidores tricantinos» que, dirigido por el autor y realizado con la colaboración, entre otros, de Daniel Galván Pascual y Nuria Gómez García, fue promovido por el Ayto. de Tres Cantos y la Dirección General de Comercio Interior de la Comunidad de Madrid. 


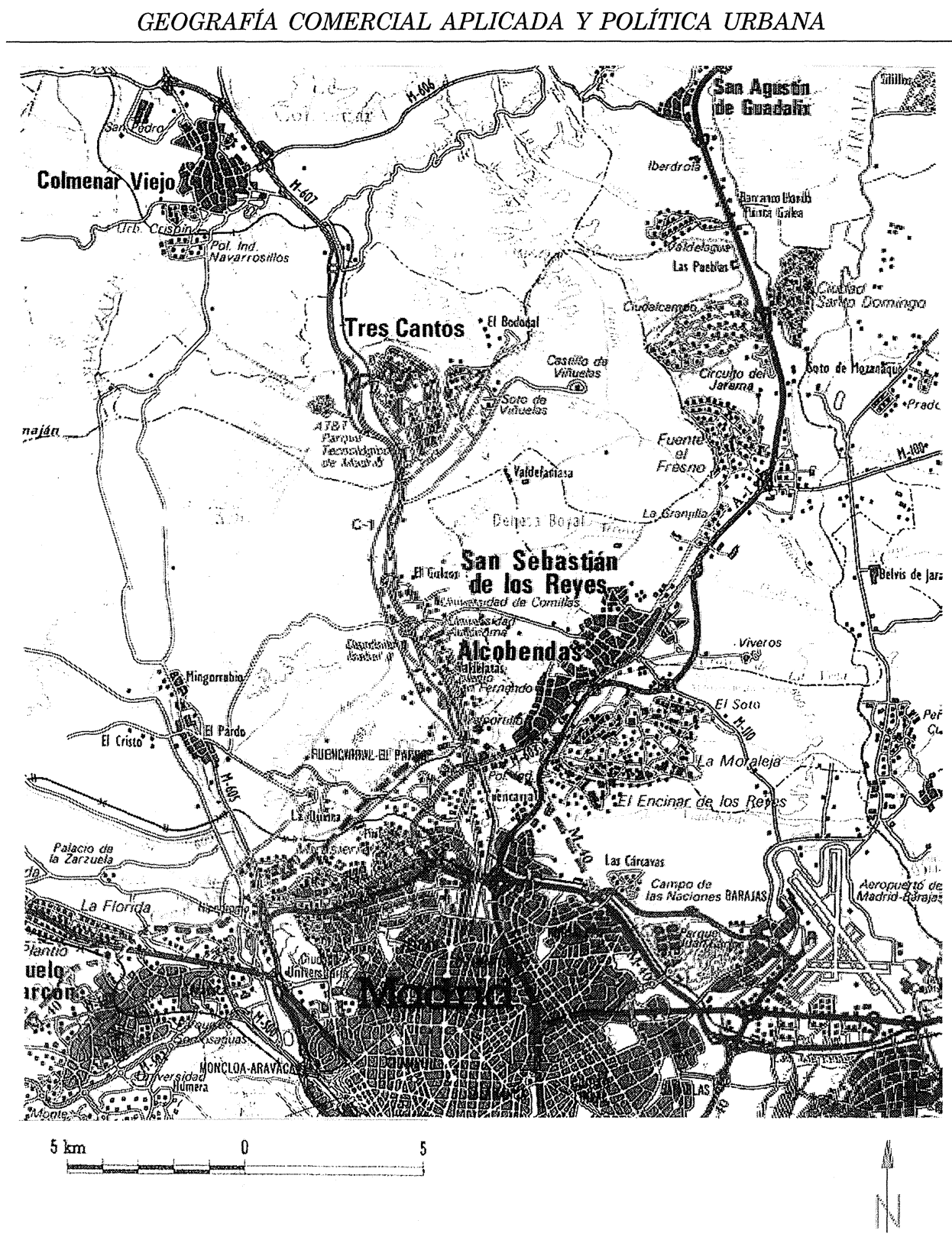

Figura 1.-La localización de Tres Cantos en el contexto metropolitano madrileño.

nica por autovía y ferrocarril (figura 1), y está adyacente a un enclave forestal de primer orden (el Monte del Pardo y el de Viñuelas). Creada como una Actuación Urbanística Urgente (ACTUR) por el Mi- 
nisterio de la Vivienda mediante un decreto-ley de 1970, se empieza a poblar hacia 1982 y en 1991 adquiere su autonomía como municipio (por segregación del de Colmenar Viejo), tras haber acogido a una cantidad importante de población y de actividades económicas, entre las que cabe destacar el Parque Tecnológico de Madrid (vid. Egea y Fernández, 1993). Todo ello le supone unas bases prometedoras y unas oportunidades innegables en la dirección hacia la que caminan las sociedades más desarrolladas y postindustriales. No obstante, su inmadurez como asentamiento y la existencia de fuertes focos competidores en su derredor, le han planteado ciertos obstáculos. En el caso de los establecimientos comerciales y de servicios a la población merece la pena reseñar que, en un radio de aproximadamente 15 minutos, los tricantinos tenían a su alcance en 1995 oportunidades como las siguientes: En Madrid el Centro Comercial Madrid-2 o La Vaguada, con el hipermercado Alcampo y Galerías Preciados (luego absorbidas por el Corte Inglés). En Alcobendas, el hipermercado Continente y el centro comercial La Gran Manzana con el hipermercado Eroski, a los que se han añadido con posterioridad tres grandes centros comerciales nuevos. En Colmenar Viejo, el centro comercial El Mirador.

Los avatares y retrasos en la cristalización de la nueva ciudad supusieron una base deficiente para el desarrollo armónico de muchas de las funciones normales de un asentamiento de su tamaño actual, en particular la comercial y de servicios al consumo. Durante los años ochenta sólo la parte N (Primera Fase) y la urbanización del Soto de Viñuelas (al E), poseían cierto desarrollo. Durante la primera mitad de los noventa se han ido cerrando las fisuras en su articulación espacial con el despegue de la parte S (Segunda Fase), que apenas era un embrión hasta entonces, y de la zona central (entre las fases primera y segunda). La figura 2 muestra una imagen de la estructura residencial de la ciudad a comienzos de 1995. La franja ondulada oriental en blanco corresponde a una de las zonas industriales.

Desde el punto de vista de su composición demográfica, posee muchas singularidades que condicionan fuertemente su papel como «mercado». La composición de edades (figura 3) exhibe el clásico perfil bimodal de las ciudades perimetropolitanas surgidas por inmigración de familias en el estadio denominado como «nido lleno» 


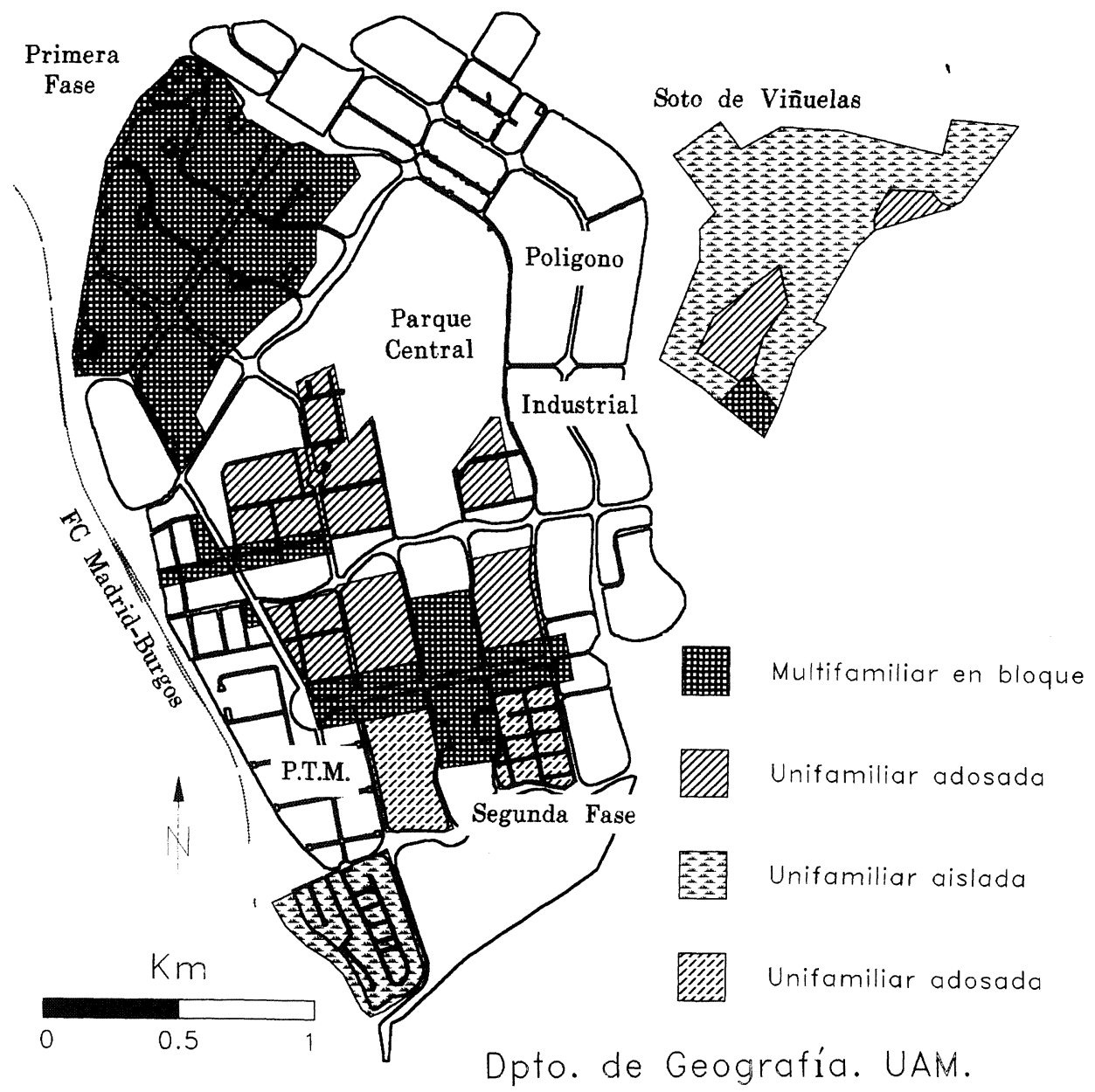

Figura 2.-Estructura residencial de Tres Cantos a principios de 1995. Paralelamente al ferrocarril, discurre la autovía. Nótese, asi mismo, el Parque Tecnológico de Madrid (PTM) al SW.

(hijos en edad infantil o adolescente). A partir tanto de datos censales (1991), como de una encuesta realizada en los hogares de la ciudad (en 353 viviendas), se revelan algunos rasgos muy especiales: una alta tasa de actividad $(64,4 \%$, superior por tanto al $52,9 \%$ de la Comunidad de Madrid, sobre todo por parte de las mujeres), un empleo fundamentalmente terciario $(74,45 \%$ de la población ocupada), una ubicación predominante de los lugares de trabajo fuera de la ciu- 


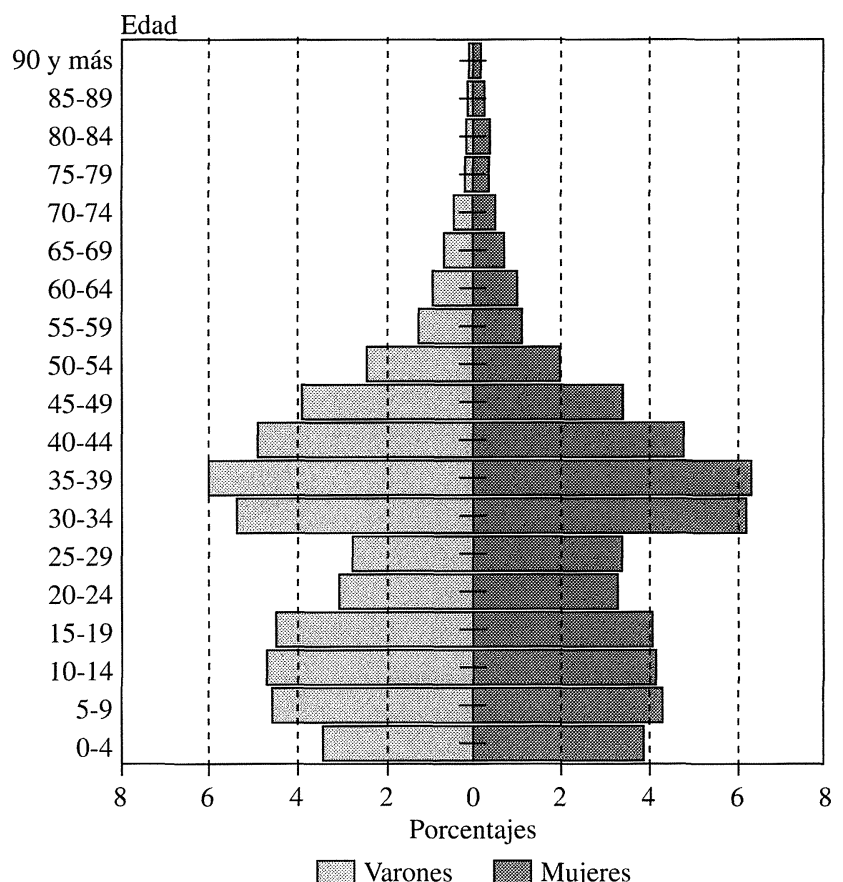

Datos provisionales

Figura 3.-Pirámide de población de Tres Cantos. Efe. Ayto. de Tres Cantos (enero de 1995).

dad $(72,3 \%$ para los varones y $39 \%$ para las mujeres, sobre todo en Madrid capital), una importantísima tasa de motorización por hogar (un $50,9 \%$ de ellos poseen dos o más coches) y una elevada instrucción $\left(25,8 \%\right.$ de la población de más de 10 años ${ }^{2}$ tiene titulación universitaria, más del doble de la cifra de la Comunidad de Madrid). Todo ello conduce a un nivel socio-económico calificable de medio y alto.

Todos los anteriores datos concitan la aparición de un síntoma bien conocido en el municipio: la evasión del poder de compra, es de-

2 La agrupación de los datos publicados ha impedido referir este dato a la población de 20 y más años, como sería recomendable. 
cir, la adquisición de bienes y servicios fuera de la localidad con el consiguiente drenaje de recursos, al margen del sector comercial instalado en ella. Ello supone desde el punto de vista del comprador unos efectos negativos, por ejemplo, más tiempo y coste de transporte que si ceteris paribus la compra se realizase en la ciudad. Pero quizá los síntomas más eficaces para catalizar el interés consistorial han venido desde el lado de la oferta, es decir, de las empresas de comercio y servicios privados para el consumo canalizadas a través de sus organizaciones representadas en la Mesa Sectorial de la Concejalía de Comercio e Industria. Dos de tales síntomas han resultado muy visibles: por un lado la pérdida de clientela, lo que reduce la rentabilidad de los negocios, por otro y, en relación con lo anterior, la elevada tasa de fracasos comerciales (establecimientos que cierran en poco tiempo). La valoración dominante entre los agentes públicos y privados era que el sistema comercial y de servicios para el consumo no funcionaba correctamente, perjudicando a intereses de los empresarios, de los consumidores y del propio Ayuntamiento.

Ante ello se avistó la realización de un estudio de diagnóstico de la situación y de sus condicionantes, para perfilar una estrategia de acción. El informe, además de considerar los aspectos urbanos, se centró prioritariamente en el análisis de las dos caras del sistema comercial: las características de la oferta y los hábitos de compra, con especial atención al comportamiento espacial de la demanda. En los dos apartados siguientes se exponen brevemente los principales hallazgos al respecto.

\section{La dotación comercial y de servicios privados para el consumo}

La información estadística para sustentar el análisis de esta parcela hubo de ser recogida, amén de los organismos habituales (Ayuntamiento, Consejería de Economía de la Comunidad de Madrid, Cámara de Comercio e Industria de Madrid, etc.), de los propios empresarios, merced a una encuesta exhaustiva realizada mediante la técnica de entrevista en 1994.

La composición del comercio y los servicios a la población, desde el punto de vista empresarial, denota el habitual predominio de las empresas pequeñas, con establecimiento único (más del $80 \%$ ) y de ti- 


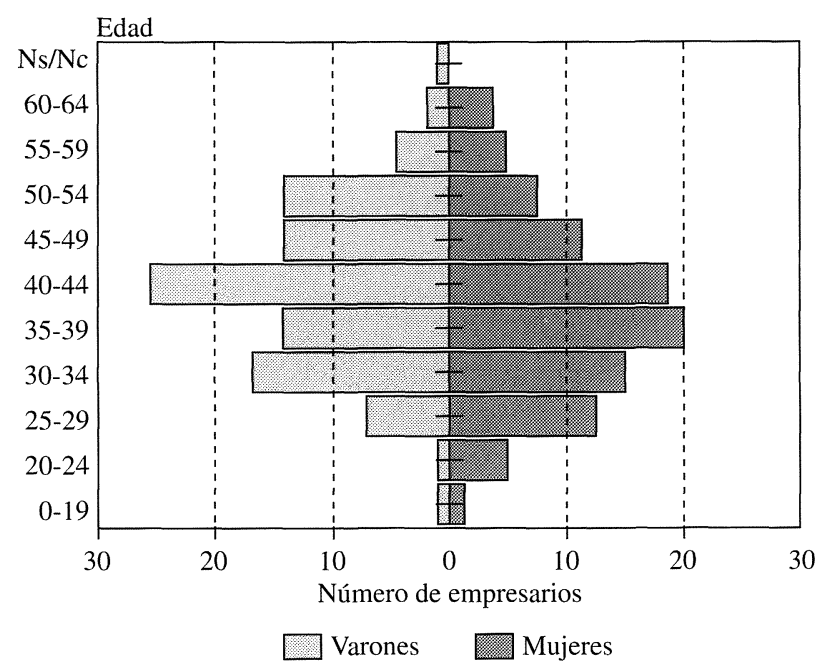

Figura 4.-Pirámide de edades de los empresarios del comercio y servicios para el consumo de Tres Cantos. Fte.: Encuesta propia, 1994.

tularidad personal, aunque este aspecto está algo menos acentuado entre los servicios.

Las formas organizativas más complejas basadas en el asociacionismo ostentan una baja penetración (14,9 por ciento entre el comercio) y así mismo las técnicas de venta más modernas no poseen tampoco una especial presencia entre el comercio, quedando por tanto terreno para mejorar en esas direcciones.

El empresariado no posee una edad elevada, siendo los intervalos modales 40-44 años para los varones y 35-39 años para las mujeres (figura 4). En cuanto al nivel de preparación, la instrucción general muestra la prevalencia de los grados de primaria y secundaria, aunque un 25,6 \% había alcanzado graduación universitaria. La formación específica (cursos ad hoc), sólo ha alcanzado a poco más de un tercio de ellos $(35,4 \%)$ siendo más baja entre los comerciantes $(27,1 \%)$ que entre los empresarios de servicios (48,1\%).

El análisis de los niveles dotacionales con un indicador clásico, pero imperfecto, como es el número de establecimientos comerciales respecto a la población (el empleo o superficie comercial habrían resultado preferibles), arroja unas cifras muy bajas cuando se confronta con otros ámbitos de referencia (cuadro T). Por tanto, a priori queda

$$
-674-
$$


un margen importante de oportunidades para incrementar la superficie ocupada por estas funciones. Los déficits en la oferta, aunque casi generales, se acentúan muy seriamente en ciertos grupos, en particular en alimentación, bebida y tabaco, y en textil y calzado. En el caso de los servicios, lo más destacable son las carencias en las actividades para el ocio y las especialidades médicas.

CuAdro 1

COMPOSICIÓN COMPARADA DEL COMERCIO (\%)

\begin{tabular}{l|c|c|c|c}
\hline & Tres Cantos & Madrid & C. Viejo + T.C. & Majadahonda \\
\cline { 2 - 5 } Alimentación, bebida, tabaco & 30.80 & 41.33 & 35.99 & 24.39 \\
Textil, calzado & 16.46 & 18.10 & 21.60 & 28.92 \\
Farmacia, drog., perfumería & 6.33 & 7.91 & 5.95 & 2.61 \\
Equipamiento del hogar & 20.68 & 14.72 & 17.21 & 23.00 \\
Automov., bicic. y accesorios & 2.53 & 2.71 & 2.82 & 3.14 \\
Carburantes y lubricantes & 0.84 & 0.23 & 0.78 & 0.52 \\
Otro comercio por menor & 20.68 & 14.14 & 14.08 & 16.90 \\
Com. mixto gran superficie & 1.69 & 0.85 & 1.56 & 0.52 \\
\hline \multicolumn{1}{c}{ Total } & 100 & 100 & 100 & 100 \\
\hline
\end{tabular}

Fuente: Elaboración propia sobre datos de encuesta y del Anuario Estadístico 1991. Volumen II. Municipios de la Comunidad de Madrid. Madrid, Comunidad de Madrid, Consejería de Economía, 1992.

N.B. Los datos de Tres Cantos van referidos a 1994. Los restantes a 1991. Los datos de Colmenar Viejo incluyen también para este último año los de Tres Cantos.

La distribución intraurbana de los establecimientos comerciales corre bastante pareja con el espacio residencial y la densidad demográfica. Sin embargo, el largo periodo durante el cual la ciudad ha estado conformada por tres zonas residenciales aisladas entre sí (Primera Fase, Segunda Fase y Soto de Viñuelas) ha impedido aprovechar el efecto de aglomeración (economías urbanas de escala) por parte de muchas de las actividades tradicionalmente calificadas como «no básicas», tales como el comercio o los servicios a la población. Los déficits dotacionales en comercio y servicios no son idénticos en las diferentes zonas de la ciudad. Si la Primera Fase y, en menor grado, la Segunda reúnen un conjunto importante de establecimientos, la Zona Central y el Soto de Viñuelas ofrecen una desdotación casi total (figuras 5 y 6 ). 


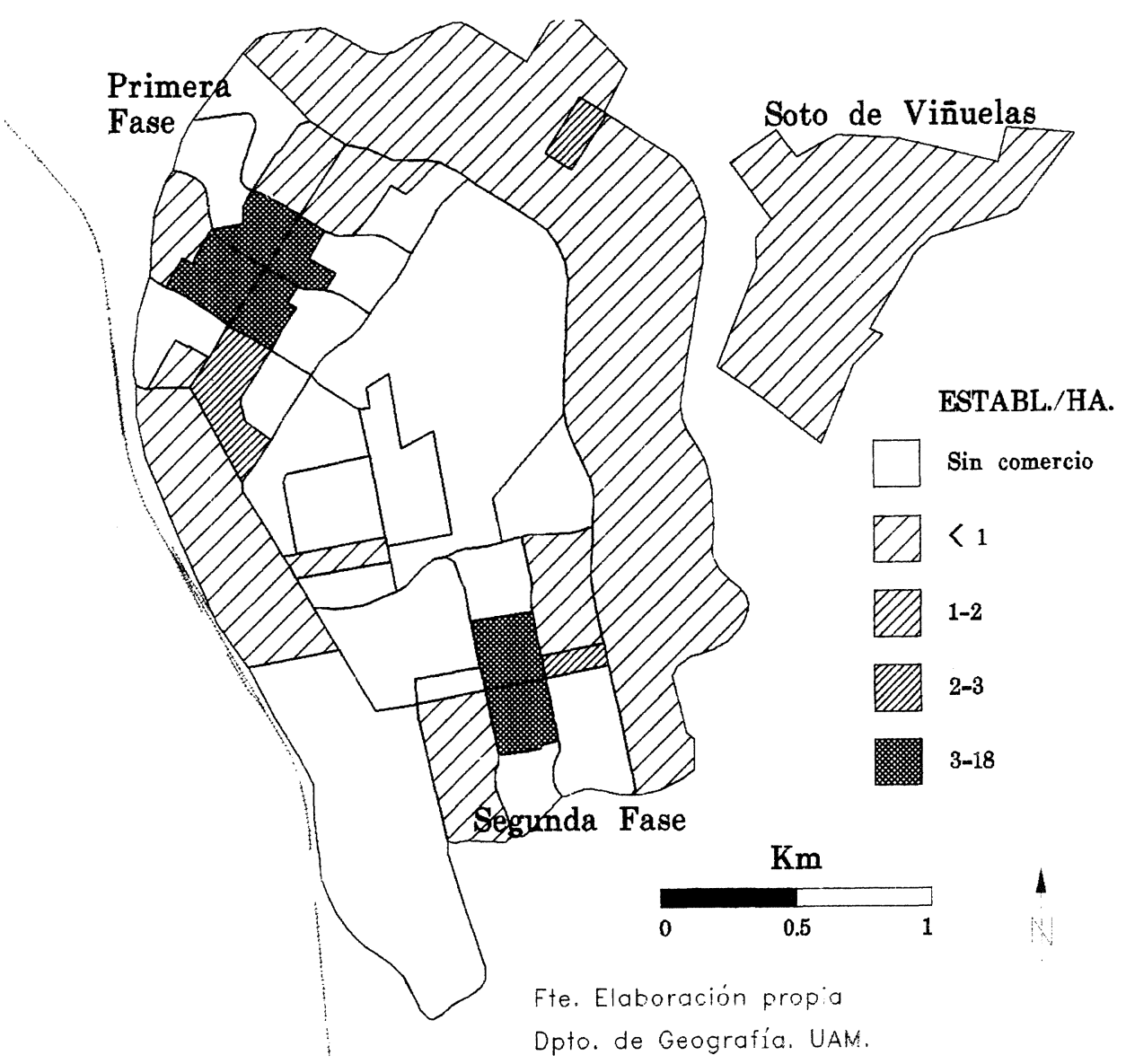

Figura 5.-Densidad intraurbana del comercio en Tres Cantos, 1994.

El diseño urbanístico de la ciudad presenta singularidades que modifican las pautas tradicionales de inserción del comercio en el espacio construido. Fruto de una dirección urbanística realizada en unas condiciones sin parangón en nuestro país la estructura de esta ciudad ofrece los siguientes rasgos dignos de ser comentados en su relación con las demandas de las implantaciones comerciales:

- Amplitud de zonas peatonales y abundancia de jardines que conducen a un debilitamiento de la visibilidad, contigüidad y coalescencia requerida por muchos establecimientos.

$$
-676-
$$




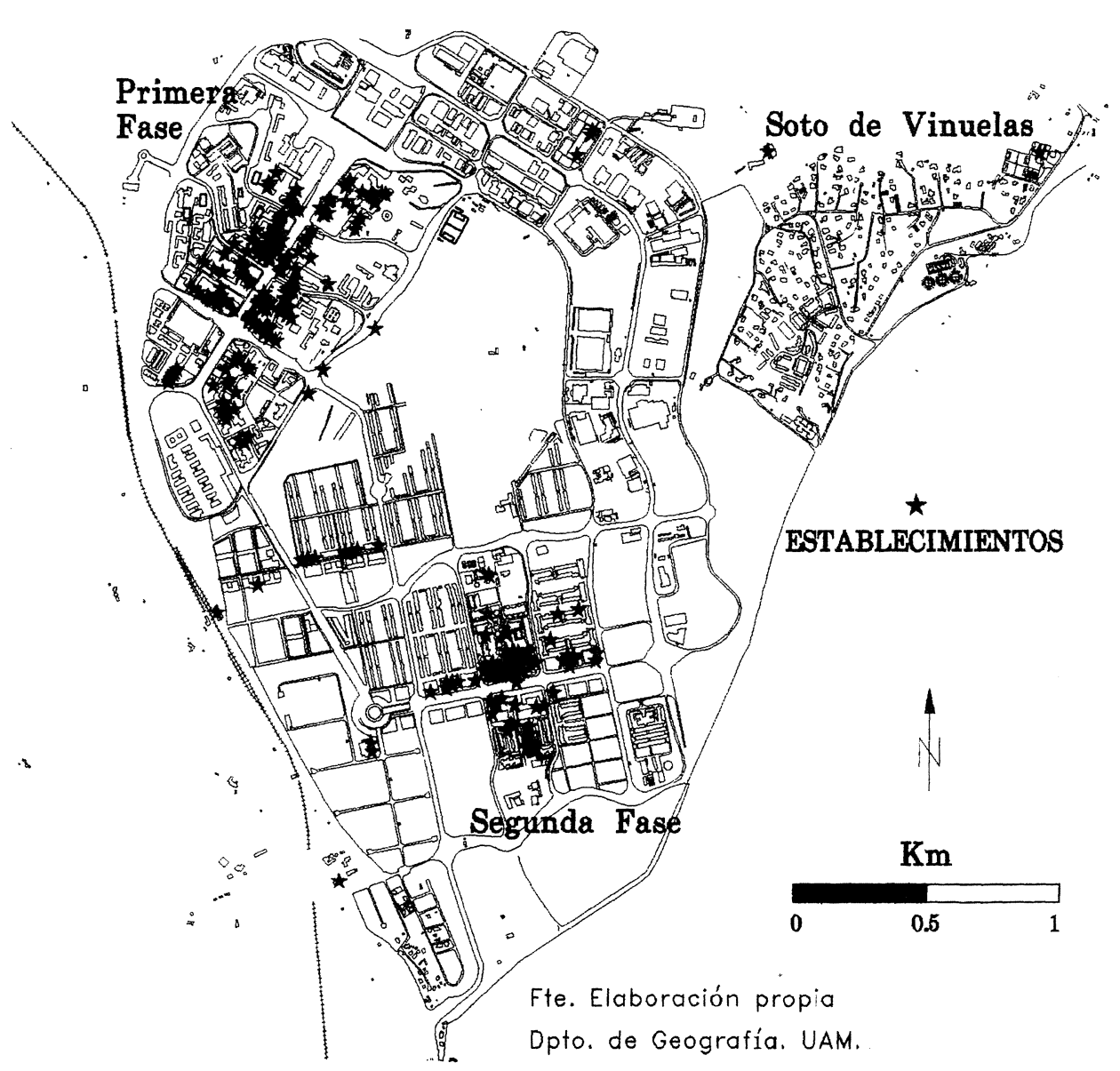

FIGURA 6.-Localización intraurbana de los servicios privados para la población en Tres Cantos, 1994

- Neto predominio de la edificación abierta, sobre todo en las zonas de edificios multifamiliares, que rompe el concepto de manzana y calle, lo que dificulta la cristalización de ejes comerciales.

- Flujos peatonales que, ante la amplitud de la configuración urbana, resultan poco canalizados, esto es, más difusos en el espacio, lo que disminuye la probabilidad de hallar emplazamientos con densa frecuentación de personas. 
- Fuerte especialización de los usos del suelo; extrema ya en las áreas de vivienda unifamiliar, de tal suerte que a veces resulta prácticamente imposible la implantación integrada de otras actividades.

- Asignación de locales para comercios y servicios a pequeños inmuebles segregados, pensados para ofrecer el primer escalón en la jerarquía de dotaciones (la unidad vecinal). La tipología de éstos existente en la ciudad es muy exigua; de hecho se puede reducir a dos grupos según que conformen o no un recinto transitable dentro del inmueble. En el primer grupo se incluirían la galería comercial (El Zoco en el Sector Pueblos que reúne 24 establecimientos) y un minúsculo centro comercial (Mercacentro, sito en Descubridores, con 15 establecimientos); del segundo existe una cifra mayor aunque su grado de ocupación es todavía muy desigual. Todo ello supone, a menudo, dificultades nuevas para trabar el tejido comercial, para definir ejes o nucleaciones de superior poder de atracción de clientela o para asegurar la visibilidad los establecimientos. De lo expuesto hay que concluir que la funcionalidad y confort de realizar las compras en un ambiente agradable y protegido de la intemperie constituye todavía una laguna a llenar en el desarrollo de la ciudad.

La indagación entre los responsables de los negocios acerca de su visión de los problemas del sector y las medidas a adoptar arrojó un abanico de aspectos dignos de ser mencionados, aunque insuficientes para dar cuenta del estado de cosas. En conjunto se constata una conciencia palpable de la seriedad de los problemas, ya que sólo en algunos grupos de actividad la situación es más despejada. De la valoración que realizan sobre diversos aspectos influyentes en su negocio merecen destacarse como más negativos los costes de los arrendamientos, la insuficiente complementariedad y variedad de la oferta, la escasa demanda y ciertos aspectos del entorno comercial y de servicios (diseño urbanístico). De forma espontánea se añadieron por los encuestados algunos otros items concernientes al excesivo peso de los «gastos, cargas fiscales y tasas». De cara a la acción, como medidas reclamadas por el lado de la oferta merecen destacarse: mejora de la señalización comercial, campañas de publicidad conjuntas, información sobre oportunidades para nuevos negocios, fomento del asociacionismo y mejoras en el entorno (zonas de aparcamiento, carga y descarga, transporte público, iluminación, etc.). 
El comportamiento de los hogares de Tres Cantos como compradores

La necesidad de desvelar las claves de ese comportamiento obligaron, ante la inexistencia de datos, a llevar a cabo un importante trabajo de campo, con el que se entrevista a una muestra representativa de 352 hogares. Ello conduciría a un error máximo de 5,11 puntos porcentuales son el $95 \%$ de nivel de confianza. El cuestionario abordaba la determinación de los lugares y tipos de establecimientos de compra o uso habitual, los factores aducidos para esa elección, los modos de compra o uso, así como una valoración de numerosos aspectos de la dotación comercial y de servicios de la ciudad.

Los hábitos de compra de los hogares muestran la diversidad esperable dada la variedad de productos y servicios que se consumen, la desigual dotación según zonas de la ciudad y la especificidad de los rasgos personales y de movilidad habitual de los tricantinos. La evasión de compras se midió con el indicador de lugar de compra habitual, aunque para los artículos alimenticios se obtuvo, así mismo, la magnitud del gasto. Los resultados con el primero de los indicadores sintéticamente fueron así:

- En los artículos de alimentación frescos hay un alto grado de compra dentro de la ciudad, si bien la escasez de oferta en ciertas zonas genera corrientes destacables entre unas y otras dentro de la misma.

- Algo similar ocurre en los productos de ferretería y de farmacia, si bien en este último caso con menos flujos internos.

- En los de alimentación no perecederos (ultramarinos, lácteos, etc.) la competencia exterior (los hipermercados y el municipio de Alcobendas) comienza a tomar fuerza.

- Algo análogo aparece en el caso de los de cosmética, si bien ahora el imán lo conforma Madrid (tiendas especializadas).

- En papelería y fotografía-óptica, si bien el mercado interior domina, Madrid es una plaza que atrae una fracción de la demanda significativa.

- En los grupos de vestido y textil, calzado, muebles y electrodomésticos se manifiesta el más intenso dominio de otros municipios (Madrid con abrumadora mayoría). 
- En juguetes y artículos deportivos, Madrid es el destino de la minoría mayoritaria, si bien Tres Cantos, y en menor grado Alcobendas, logran cierta cuota de mercado. En relojería y joyería las proporciones entre destinos son parecidas si bien Alcobendas decae fuertemente.

En el terreno de los servicios privados para el consumo aflora de nuevo una gran diversidad:

- En bares y cafeterías, instalaciones deportivas, estudios fotográficos, lavanderías-tintorerías y peluquerías se elige predominantemente Tres Cantos.

- Para los restaurantes domina como destino Madrid, con una parte significativa también para Tres Cantos y otros lugares.

- Para las reparaciones (del hogar, de vehículos) se recurre en primer lugar a los establecimientos de la propia ciudad y en segundo lugar a Madrid.

- Para espectáculos el municipio que se beneficia totalmente es Madrid.

- Para los servicios de educación privados prevalece Tres Cantos, si bien Madrid, goza de cierto predicamento.

- Algo análogo sucede en el uso de la medicina privada, general u odontológica. Sin embargo en el campo de las especialidades, la atracción de Madrid le encumbra al liderazgo.

Mediante el uso del segundo indicador, para el grupo de gasto en alimentación en conjunto, la evasión fuera de Tres Cantos se pudo cifrar semanalmente en 6.089 ptas. de media por hogar. La fracción de ese gasto evadido variaba mucho por hogares tal como muestra la figura 7, pero importa retener dos datos: por un lado que la media de los porcentajes de evasión se cifraba en 36,4 , y por otro que un 41,2 por ciento de los hogares ejecutaba fuera de la ciudad más de la mitad de su presupuesto semanal en alimentación.

Las razones más esgrimidas por los hogares a la hora de justificar las compras fuera de Tres Cantos son, con mucha diferencia, dos: el surtido y variedad de productos y los precios, aspectos en los que la ciudad resulta muy mal parada frente al exterior, a tenor la version expresada por los vecinos. Pese a todo la apreciación de los con- 


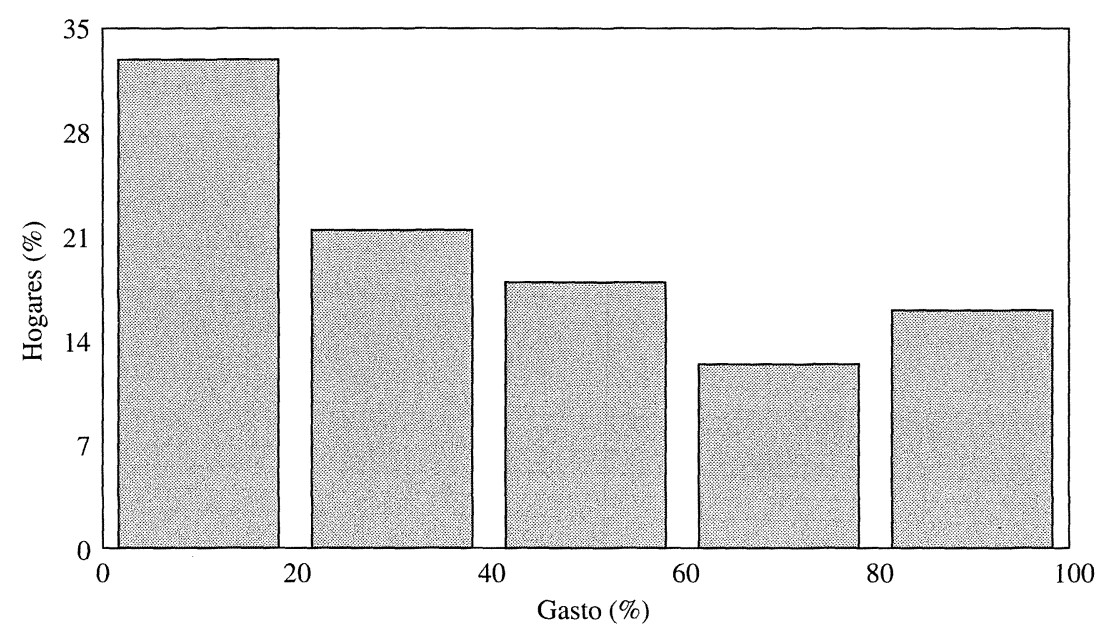

Figura 7.-Distribución de los hogares tricantinos según el porcentaje de gesto semanal que realizan en alimentación fuera de la ciudad. Fte.: Encuesta propia, 1994.

sumidores es que sus compras en la ciudad se van incrementando paulatinamente. Ese uso de los establecimientos de la ciudad se sustenta sobre todo en consideraciones, por este orden, de cercanía, comodidad y trato personalizado.

Las valoraciones que sobre la oferta comercial de la ciudad emiten los responsables de las compras es bastante critica en cuanto al nivel de precios y al número y variedad de comercios. Si es mejor en lo atingente al entorno comercial, horarios, profesionalidad de los vendedores y ubicación y acceso a los establecimientos, si bien persisten fracciones notables de descontento en los diferentes aspectos mencionados. Ante la tesitura de explicitar las demandas más sentidas por los responsables de las compras, éstas se orientan en varias direcciones, destacando globalmente:

- Establecimientos de cultura y ocio, vestido y calzado.

- Una "gran superficie», según el modelo que se ha popularizado en nuestras periferias urbanas.

- Apertura los sábados tarde, posibilidad que podría resultar muy positiva, ya que cuenta con la ventaja de la presencia en la ciudad del grueso de sus habitantes. 
- Eventualmente y por zonas surgen demandas especificas a tener presentes, como por ejemplo mejoras en el transporte público interior.

Un análisis de los factores condicionantes de la elección del lugar de compra fue ejecutado adicionalmente mediante el recurso a técnicas estadísticas bivariadas y multivariantes, aunque su extensión obliga a posponer para otro lugar su presentación.

\section{Propuestas y recomendaciones}

Fruto del amplio diagnóstico y análisis explicativo desarrollado previamente se avistó a la concreción de un conjunto de líneas de actuación que, sustentadas en unos principios y objetivos, concernían a diversas facetas del sector. A efectos expositivos, se agrupan en otros tantos apartados.

Criterios y objetivos.-Cuatro fueron las bases que dan fundamento a las recomendaciones:

A) Consolidación de una oferta comercial lo suficientemente amplia, viable y ajustada como para satisfacer in situ el mayor porcentaje posible de la demanda de los consumidores, reduciendo drásticamente la evasión de compras y la generación de viajes por desdotación o desajuste de la oferta local.

B) Conformar una distribución intraurbana de la oferta más equilibrada respecto a la estructura de la ciudad y acorde con los rasgos de la demanda.

C) Minimizar algunos problemas crónicos, unos inherentes a la competitividad y gestión del pequeño comercio y otros asociados a la singularidad urbana de Tres Cantos.

D) Mejorar las bases sobre las que tradicionalmente se ha apoyado la toma de decisiones estratégicas por parte de los proveedores de bienes y servicios, en aras de reducir los fracasos de iniciativas empresariales.

Recomendaciones sectoriales.-Dos propuestas globales, que se despliegan en otras más detalladas, parecieron prioritarias:

$$
-682-
$$


A) En primer lugar, el fomento de formas de organización empresarial y técnicas de venta más avanzadas y acordes con la especificidad de la composición y estilos de vida de la población de la ciudad. En particular:

- Potenciación del asociacionismo empresarial, tanto en redes interurbanas, como por zonas en Tres Cantos como vía para incrementar la competitividad y la modernización.

- Uso más amplio de técnicas de marketing adaptadas al pequeño comercio tales que mejoren así mismo la competitividad: reducción de precios, ampliación de servicios al cliente (teniendo especialmente en cuenta la existencia de ciertas zonas de viviendas unifamiliares y aceptable nivel de renta), etc.

B) Y en segundo, la orientación de las iniciativas de mayor dotación hacia los grupos de actividad con demostrado déficit, en particular: a) Alimentación, bebida y tabaco, b) textil y calzado, c) farmacia, d) ocio, y e) especialidades médicas.

Recomendaciones sobre organizacion urbana.-Habida cuenta de que la propia inserción urbana de la función de distribución es responsable de no pocos problemas en general, y en el caso de Tres Cantos con mayor agudeza, se postularon las siguientes indicaciones:

A) Fomentar la cristalización de ejes o zonas de comercio tanto de nivel de barrio, como para el conjunto urbano de modo que actúen de focos de atracción y retención de clientela en función de la diversidad y complementariedad que deben ofrecer. En particular:

1) Segunda Fase: Fortalecer la zona comercial y de servicios en torno al eje de Avda. de Viñuelas para acceder a nivel de centro de barrio.

2) Zona central: Instigar la dotación de comercio de dos niveles:

a) Comercio y servicios de barrio, que satisfaga la demanda de las colonias inmediatas de viviendas adosadas.

b) Comercio y servicios de mayor rango, propios de un centro de ciudad. 
Diseñar meticulosamente el tamaño y composición del futuro centro de ocio y comercio a la luz del modelo futuro de ciudad, de la dotación comercial intraurbana actual y deseable y de la competencia exterior.

3) Soto de Viñuelas: Orientar una dotación puntual de comercio de bienes de consumo frecuente, teniendo muy en cuenta la peculiaridad urbanística y social de la zona, a efectos de los estándares y servicios ofrecidos por los establecimientos.

B) Mejorar de forma eficaz la visibilidad y conocimiento por la demanda de la oferta y de los establecimientos comerciales. En este sentido se insiste en la necesidad de paliar las consecuencias del diseño urbanístico de la ciudad mediante, entre otras medidas, una señalización de la ubicación de establecimientos, que a la vez sea compatible con la estética urbana.

C) Abordar ciertas mejoras en los principales focos comerciales (Primera y Segunda Fase que afectan a zonas de aparcamiento, carga y descarga, iluminación, transporte público (recientemente adoptadas), etc.

Recomendaciones para apoyo a las empresas.-En el terreno de las iniciativas que más directamente implican a las empresas del sector se propusieron:

A) Fomento del nivel de cualificación técnico-empresarial, difundiendo entre otros aspectos:

- Las ventajas de formas más modernas de gestión y organización empresarial (por ejemplo el asociacionismo).

- Las ventajas de la evaluación de oportunidades y riesgos mediante métodos más rigurosos, como el geomarketing.

- Las ventajas de medidas conjuntas y colaboración en distintos terrenos de la gestión empresarial, etc.

B) Implicar eficazmente a los comerciantes y empresarios de servicios, a través por ejemplo de sus asociaciones, en la promoción y ejecución de medidas de apoyo a sus actividades. 
C) Potenciar la colaboración del Ayuntamiento con los empresarios del comercio y servicios en varios campos, considerando, entre otros aspectos, los siguientes:

- Información sobre iniciativas del Ayuntamiento y ayudas públicas al comercio y desarrollo local.

- Patrocinio selectivo de medidas conjuntas de promoción.

- Asesoramiento de oportunidades y de competencia interior y exterior (entorno geográfico próximo).

- Seguimiento e información sobre hábitos de la demanda y tendencias del consumo.

D) Promover la implantación de un Sistema de Información Geográfico-Comercial (SIGECO) para soporte de las iniciativas públicas y de las estrategias empresariales. Por un lado, la administración local mejoraría ostensiblemente su capacidad de coordinación interna y de adopción de politicas. Por otro, los empresarios podrían obtener de forma muy ágil y visual información básica sobre el sistema de provisión de bienes y servicios a la población (los emplazamientos más idóneos, la ubicación y rasgos de competidores, la identificación y localización de los segmentos de la demanda buscados, etc.).

Recomendaciones de estudios técnicos.-Finalmente, y dada la dinámica y tensiones que la distribución minorista de bienes y servicios afronta en las presentes coordenadas, se postularon otras tres iniciativas adicionales:

A) Revisar el diseño futuro de la estructura comercial a la luz de las previsiones del planeamiento urbano y de crecimiento de la ciudad.

B) Analizar especialmente el previsto centro de ocio y comercio y determinar su impacto en variados aspectos: red viaria, aparcamiento y transporte, economía (hacienda local, empresas del comercio y los servicios), empleo, medio ambiente urbano, comportamientos sociales, seguridad ciudadana, etc.

C) Avistar a un seguimiento de las medidas adoptadas a través de estudios periódicos que analicen evolutivamente tanto los rasgos y estrategias de la oferta, como el comportamiento y actitudes de la demanda. 


\section{Consideraciones finales}

Las diversas transformaciones que la distribución minorista de bienes y servicios, el comportamiento de los consumidores y las estructuras metropolitanas están atravesando, generan un contexto nuevo y en continua inestabilidad, lo que desemboca en las tensiones y desajustes propios de las transiciones en cualquier sistema. La necesidad de adaptación y re-acomodo casi permanente que se generan están despertando la conciencia de los agentes que ostentan responsabilidades tanto en el campo empresarial, como en el público (la Administración) de avistar a decisiones estratégicas ante las que, en muchos casos (sobre todo las empresas pequeñas y los organismos municipales), se hallan bastantes desvalidos, por el cúmulo de incertidumbres y desconocimiento acerca de las raíces de los problemas y las opciones realistas de actuación. Ello está brindando oportunidades, que todavía han de ampliarse mediante «marketing» de la ciencia, de llevar a cabo estudios de geografía aplicada en ese terreno, de tal suerte que, combinando el rigor del análisis y el realismo de las propuestas, ayuden a una toma de decisiones mejor fundada. El caso expuesto escuetamente en este artículo se ha presentado con la intención de divulgar una experiencia positiva que puede resultar ilustrativa y fértil para la orientación futura de geógrafos profesionales. La perspectiva propia de nuestra disciplina, atenta no sólo a los aspectos económicos, sino también a los urbanos o espaciales, conlleva una integración sin par de los elementos relevantes, hecho que puede aportar un valor añadido difícilmente igualable por otras.

En lo que concierne al caso estudiado conviene subrayar que su problemática resulta, en ciertos aspectos, representativa de esos municipios de las periferias metropolitanas que, desarrollados al calor de la corriente suburbanizadora poseen un tejido comercial todavía débil e insuficiente para la demanda local en ascenso; pero al mismo tiempo esta demanda no ha alcanzado los umbrales mínimos para hacer viable la implantación de una gran superficie comercial. Los atributos de la demanda y su conducta espacial la aproximan bastante al modelo de «consumidor geográficamente móvil» cuya conquista por parte de la oferta local y su retención en el municipio suponen un fuerte desafio, tanto para la ciudad, como para los empresarios del sector. El poder de la competencia, materializada en la dotación ubi- 
cada en otros municipios adyacentes, que no solo es empresarialmente más competitiva, sino también más amplia, exige que estos focos comerciales secundarios adopten un compromiso permanente de análisis de estrategias económico-territoriales, involucrando activamente tanto a los pequeños empresarios, coma a las autoridades locales. El comienzo de la resolución de problemas pasa, a nuestro juicio, por una etapa previa de mentalización y asunción, por parte de ambos agentes, de la inexorable necesidad de pensar y actuar coordinada y convergentemente. En el municipio en cuestión esa premisa comienza ya a ser una realidad.

\section{BIBLIOGRAFÍA}

Beaumont, J. (1991): «GIS and market analysis» en Maguire, D., Goodchild, M. y Rhind, D. (ed.): Geographical information systems. Harlow, Longman, v. 2, pp. 139-151.

Birkin, M., et al. (1996): Intelligent GIS. Location decisions and strategic planning. Cambridge, Geoinformation International.

Egea, M. y Fernandez, D. (1993): Tres Cantos. Veinte años de historia (1971-1991). Madrid, Iniciativas y Publicaciones.

Jones, K. y Simmons, J. (1990): The retail environment. Londres, Routledge.

Longley, P. y Clarke, G. (1995): GIS for business and service planning. Cambridge, Geoinformation International.

Merenne-Schoumaker, B. y Browet, A. (1996): Localisation du magasing. Guide pratique. Bruselas, Comité Belge de la Distribution, 3. ${ }^{\mathrm{a}}$ ed.

Moreno, A. (1995): «Planificación y gestión de servicios a la población desde la perspectiva territorial: algunas propuestas metodológicas», Boletín de la Asociación de Geógrafos Españoles, 20, pp. 115-134.

- (1995, Dir.): El equipamiento comercial y la evasión del poder de compra de los consumidores tricantinos. Ayuntamiento de Tres Cantos (Madrid) y Dirección General de Comercio Interior de la Comunidad de Madrid (policopiado).

Moreno, A.; Gómez García, N. y Galván Pascual, D. (1995): «Los SIG como soporte de la política y gestión municipal en el campo del comercio minorista. Un estudio de caso». Cambios regionales a finales del siglo XX. XIV Congreso Nacional de Geografía, Salamanca, Asociación de Geógrafos Españoles y Univ. de Salamanca, pp. 425-429.

RESUMEN: Las tensiones originadas por los cambios urbanos y en la distribución comercial y de servicios están haciendo brotar la necesidad del asesoramiento por parte de expertos, tanto a las empresas, coma a los organismos públicos para adoptar decisiones estratégicas. En este articulo se resumen las lineas maestras de un estudio que se orientó a proveer de un diagnostico de la situación y de propuestas, en materia comercial, para informar la politica municipal de la ciudad nueva de Tres Cantos, sita en el área metropolitana de Madrid.

Palabras Clave: Geografía aplicada, geografía del comercio, política urbana. 
AвstRACT: The tensions emerged from urban, retail and services distribution changes are fostering the need of experts' advice not only to private firms, but also to public agencies to tackle strategic decisions. In this paper it is presented a synthesis of a larger study focused to provide a situation diagnostic and proposals, concerning the retail sector, to enlighten the urban policy of Tres Cantos, a new town in the Madrid metropolitan area.

KEYwoRDs: Applied geography, retail geography, urban policy.

RésumÉ: Les tensions dues aux changements urbains, commerciales et des services ont stimulé le besoin de conseil des experts aux entreprises privées et aussi aux organismes publiques pour orienter ses décisions stratégiques. Dans cet article on expose le contenu essentiel d'un étude plus ample réalisé pour procurer un diagnostique de la situation actuelle et des propositions, concernant au secteur commercial, pour orienter la politique communale de la ville nouvelle de Tres Cantos, appartenant a l'aire metropolitaine de Madrid.

MOTS-CLÉ: Géographie appliquée, géographie commerciale, politiques urbaines. 